\title{
BMJ Open Gene expression profiling in persons with multiple chemical sensitivity before and after a controlled n-butanol exposure session
}

Thomas M Dantoft, ${ }^{1,2,3}$ Sine Skovbjerg, ${ }^{3}$ Linus Andersson, ${ }^{4,5}$ Anna-Sara Claeson, ${ }^{4}$ Kaare Engkilde, ${ }^{6}$ Nina Lind, ${ }^{4,7}$ Steven Nordin, ${ }^{4}$ Lars I Hellgren ${ }^{2}$

To cite: Dantoft TM, Skovbjerg S, Andersson L, et al. Gene expression profiling in persons with multiple chemical sensitivity before and after a controlled n-butanol exposure session. BMJ Open 2017;7:e013879. doi:10.1136/bmjopen-2016013879

- Prepublication history and additional material is available. To view please visit the journal (http://dx.doi.org/ 10.1136/bmjopen-2016013879)

Received 16 August 2016 Revised 28 December 2016 Accepted 16 January 2017

CrossMark

For numbered affiliations see end of article.

Correspondence to Dr Thomas M Dantoft; thomas.meinertz.dantoft@ regionh.dk

\section{ABSTRACT}

Objectives: To investigate the pathophysiological pathways leading to symptoms elicitation in multiple chemical sensitivity (MCS) by comparing gene expression in MCS participants and healthy controls before and after a chemical exposure optimised to cause symptoms among MCS participants.

The first hypothesis was that unexposed and symptom-free MCS participants have similar gene expression patterns to controls and a second hypothesis that MCS participants can be separated from controls based on differential gene expression upon a controlled n-butanol exposure.

Design: Participants were exposed to $3.7 \mathrm{ppm}$ n-butanol while seated in a windowed exposure chamber for $60 \mathrm{~min}$. A total of 26 genes involved in biochemical pathways found in the literature have been proposed to play a role in the pathogenesis of MCS and other functional somatic syndromes were selected. Expression levels were compared between MCS and controls before, within $15 \mathrm{~min}$ after being exposed to and 4 hours after the exposure.

Settings: Participants suffering from MCS and healthy controls were recruited through advertisement at public places and in a local newspaper.

Participants: 36 participants who considered themselves sensitive were prescreened for eligibility. 18 sensitive persons fulfilling the criteria for MCS were enrolled together with 18 healthy controls.

Outcome measures: 17 genes showed sufficient transcriptional level for analysis. Group comparisons were conducted for each gene at the 3 times points and for the computed area under the curve (AUC) expression levels.

Results: MCS participants and controls displayed similar gene expression levels both at baseline and after the exposure and the computed AUC values were likewise comparable between the 2 groups. The intragroup variation in expression levels among MCS participants was noticeably greater than the controls.

Conclusions: MCS participants and controls have similar gene expression levels at baseline and it was not possible to separate MCS participants from controls based on gene expression measured after the exposure.
Strengths and limitations of this study

- This is the first study comparing gene expression levels between participants with multiple chemical sensitivity (MCS) and healthy controls before and upon a chemical exposure session optimised to trigger MCS characteristic symptom among MCS participants.

- The exposure sessions ability to segregate MCS participants from controls based on a phenotypic response had previously been verified, including increased symptoms levels, higher than normal pulse rate and lower than normal pulse rate variability associated with the MCS group.

- Genes included in the study were preselected based on their possible role in regulation of characteristic symptoms of MCS, their involvement in the physiological response observed during the exposure session, or because of their involvement in the proposed biological explanatory models of MCS.

- The limited number of study participants enrolled does represent a weakness of the study and any follow-up studies would benefit from having a larger and more representative study populations.

- MCS participants represent a fairly heterogeneous population in terms of which odours that cause symptom elicitation as well as the symptoms reported, and this intragroup variability observed among MCS participants only constitutes a statistical challenge for the group comparisons performed.

\section{INTRODUCTION}

Chemical intolerance (CI) towards everyday chemicals, such as fragranced products, freshly printed papers or magazines, car exhausts or new furniture is reported by a substantial proportion of the general adult population. ${ }^{1-5}$ A subgroup with CI reports severely debilitating symptoms when exposed to these airborne chemicals, often with negative impact on social and occupational life and reduction in overall life quality. ${ }^{6-8}$ This 
severe form of CI is commonly referred to as multiple chemical sensitivity (MCS). ${ }^{8-11}$ The prevalence of MCS in the adult population has been reported in the ranges from $0.5 \%$ to $6.3 \%$, with a higher prevalence in women compared with men. ${ }^{1-4}{ }^{12-14}$ Symptoms from the central nervous system (CNS), for example, headache, dizziness or fatigue, are considered mandatory in most definitions of MCS, ${ }^{10}$ often in combination with one or several nonspecific symptoms from other organ systems including the mucosa/respiratory tract, musculoskeletal system and the gastrointestinal tract. ${ }^{310}{ }^{15}$ How to define and isolate cases with MCS is an ongoing challenge both clinically and in terms of research and variable case definitions have been proposed and applied across the scientific literature, ${ }^{8}$ most prominently being the 1999 US Consensus Criteria, ${ }^{9}$ later extended with clarifications suggested by Lacour and colleagues in 2005. ${ }^{9}{ }^{10}$ However, considering the complexity of MCS it may not even be possible to compose a single definition that is sensitive and specific enough to cover both diagnostics and various research and epidemiological purposes. ${ }^{8} 1617$

Why some individuals develop MCS also remains disputed and the nature as well as the scale of symptoms experienced cannot be explained by traditional toxicological dose-response relationships. ${ }^{15}$ Multiple causative modes of action have been suggested to explain the disease mechanisms behind MCS, encompassing both physiological and psychological processes. ${ }^{11}{ }^{18-22}$ However, although some clinical evidence in support of several suggested pathophysiological models is available, no conclusions can be drawn based on current knowledge. $^{8} 23$ Consequently, participants with MCS are offered insufficient healthcare solutions and experience being met with doubt or limited understanding of their condition by healthcare professionals, the social welfare system and the society in general. ${ }^{8}{ }^{24-26}$ It is therefore essential that a deeper knowledge about the pathogenic pathways leading to symptoms elicitation in participants with MCS is being generated. Gene expression profiling is a recognised and reliable technique that can be used to quantitatively track functional gene expression patterns under varying environmental and experimental conditions, thereby providing novel information about the biochemical pathways activation at a specific time point (TP). It has yet to be applied in studies of MCS, but the technique has recently shown promising results in the detection of potential biomarkers in chronic fatigue syndrome, ${ }^{27-29} \mathrm{a}$ disorder that share many similarities with MCS.

In the present study, we used a similar approach, ${ }^{27-29}$ to investigate whether exposure to a symptom-eliciting chemical (n-butanol) could provoke changes in transcription levels of selected genes in leucocytes from MCS participantscompared with healthy controls, indicative of differential transcriptional regulation.

The ability to segregate MCS participantsfrom controls based on the phenotypic responses to the exposure session have previously been described, ${ }^{30}$ and we have also verified in the same individuals that localised upper airway inflammation is not a part of the pathology, suggesting that symptom elicitation in MCS has to be driven by systemic mechanisms. ${ }^{31}$ We expected this to be manifested as changes in gene transcription rates, and that differences in this response could indicate pathways involved in the MSC phenotype. As genetic studies in MCS so far have been inconclusive, ${ }^{8}$ and this being the first gene expression profiling study focusing on MCS, it was conducted as an explorative study. We chose to measure gene expression in leucocytes isolated from peripheral blood samples, because peripheral leucocytes are easily available, they have already been used to identify gene expressional changes in patients with chronic fatigue syndrome upon symptom elicitation, ${ }^{27-29}$ and because numerous studies of MCS and other functional somatic syndromes (FSS) have found indications of inflammatory abnormalities in these conditions. ${ }^{8} 24$ 32-34

Based on available knowledge in FSS in general and MCS in particular, 26 genes were selected. The genes belonged to the following regulatory categories: genes involved in immune regulation, the physiological stress response, sensory detection and enzymes of the sphingosine-1-phosphate pathway.

The following two hypotheses were tested: (1) unexposed MCS participants and healthy controls will show similar expression of the include genes of interest, and (2) upon a controlled n-butanol exposure, MCS participants and healthy controls can be distinguished on group levels based on differential gene expression patterns.

\section{MATERIAL AND METHODS \\ Study population}

Participants suffering from MCS and healthy controls were recruited through advertisement at public places and in a local newspaper covering the Västerbotten County in Sweden. Exclusion criteria were smoking, pregnancy, current breast feeding and a diagnosis of fibromyalgia, chronic fatigue syndrome or irritable bowel syndrome given by a physician. An additional exclusion criterion included anosmia, and all participants were prior to the exposure screened for this condition using a $0.44 \% \mathrm{v} / \mathrm{v}(336 \mathrm{ppm})$ concentration of n-butanol $(99 \%$, Merck) of the Connecticut Chemosensory Clinical Research Center Threshold Test. ${ }^{35}$

A total of 36 participants who considered themselves especially sensitive were contacted by phone and prescreened for eligibility using the US Consensus Criteria for $\mathrm{MCS}^{9}$ and the revisions suggested by Lacour et $a l^{10}$ which were operationalised as follows: (1) symptoms for at least 6 months; (2) symptoms occur in response to exposure to low levels of chemicals that do not induce symptoms in other participants who are exposed to the same levels; (3) symptoms occur when exposed, and lessen or resolve when the symptom-triggering exposure is removed; (4) symptoms are elicited by at least two 
unrelated chemical substances; (5) presence of at least one symptom from the CNS (eg, headache, fatigue, dizziness, memory problems, concentration difficulties or tiredness) and one symptom from another organ system; (6) symptoms that cause significant impairment in daily life, either in social, recreational, occupational, educational or economic situations (confirmed by the score on the Chemical Sensitivity Scale, ${ }^{36}$ table 2 ). A total of 18 participants (16 women, 2 men) fulfilled the MCS criteria and were included in the study.

Eighteen participants (14 women, 4 men) were recruited as age-matched and sex-matched healthy controls. The controls did not fulfil any of the study criteria for MCS, and reported no avoidance behaviour, annoyance or symptoms attributed to low-level chemical exposure. None of the participants in the control group shared housing with an MCS affected individual or had any close relative, that is, parent, grandparent, sibling or child with MCS.

\section{Exposure chamber and exposure procedure}

The exposure procedure and chamber designed used in this study is described in more detail in Andersson et a $l^{00}$ and Andersson $e t a .^{37}$ In brief, participants were exposed to n-butanol (99.4\% J.T. Baker) while seated in a windowed exposure chamber. The exposure chamber had a volume of $2.7 \mathrm{~m}^{3}$ (height: $200 \mathrm{~cm}$, width: $90 \mathrm{~cm}$, depth: $150 \mathrm{~cm}$ ) and the exposure concentration of $n$-butanol was $11.5 \mathrm{mg} /$ $\mathrm{m}^{3}$ (3.7 ppm). The odorant $\mathrm{n}$-butanol was chosen for the exposure procedure based on a pilot test in which MCS sufferers judged the compound to be symptom-eliciting and because it had been used successfully in previous challenge studies with MCS participants. ${ }^{37}$ The concentration of n-butanol was clearly detectable (above the olfactory threshold $0.012 \mathrm{mg} / \mathrm{m}^{3} 39$ ), but well below its threshold for sensory irritation $\left(75 \mathrm{mg} / \mathrm{m}^{340}\right)$.

Unknown to the participants, no odorant was delivered into the exposure chamber during the first $10 \mathrm{~min}$ of testing (figure 1). After this initial period of blank exposure, n-butanol was released into the chamber and reached its peak concentration in about $8 \mathrm{~min}$ later (figure 1). The concentration remained at this peak level for the rest of the session (42 min). The temperature and relative humidity inside the chamber were continuously monitored during the exposures and the mean temperature was $22^{\circ} \mathrm{C}\left( \pm 1^{\circ} \mathrm{C}\right)$ and the relative humidity was $16 \%( \pm 2 \%)$, same as the concurrent humidity outside the chamber.

At baseline and at regular intervals during the exposure session, the participants rated the perceived intensity and valence as well as the level of possible symptoms. Additionally, autonomic recordings of breathing rate, tonic electrodermal activity, pulse rate and pulse rate variability were obtained at baseline and periodically during the exposure. A more detailed description of the methodology used for collection of self-reported ratings and autonomic recordings has been published separately in Andersson $e t a l^{30}$ verifying the experimental setup's capability to elicit characteristic MCS symptoms in the MCS group and to produce the anticipated group differences.

\section{Sample collection}

Venous blood of $8 \mathrm{~mL}$ were collected in anticoagulant $\mathrm{Na}_{2}$-EDTA tubes (Greiner Bio-One, Kremsmünster, Austria) at three TPs; within 30 min prior to the exposure (TP1), within $15 \mathrm{~min}$ postexposure (TP2) and 4 hours after the exposure session had been terminated (TP3)outlined in figure 1 . All blood sampling and analyses were performed by personnel blinded to group affiliation. Blood samples were immediately centrifuged at $1500 \mathrm{~g}$ for 10 min without brake and the buffy coat layer was carefully collected in $1.2 \mathrm{~mL}$ RNAlater solution (Life Technologies RNA stabilisation reagent) according to the manufacturer's instructions and stored at $-80^{\circ} \mathrm{C}$ until RNA extraction.

\section{RNA extraction and gene expression analysis}

RNA isolation, cDNA synthesis and gene expression analysis were conducted by SABioscience service core laboratories (SABioscience/Qiagen, Hilden, Germany), using the protocols described below.

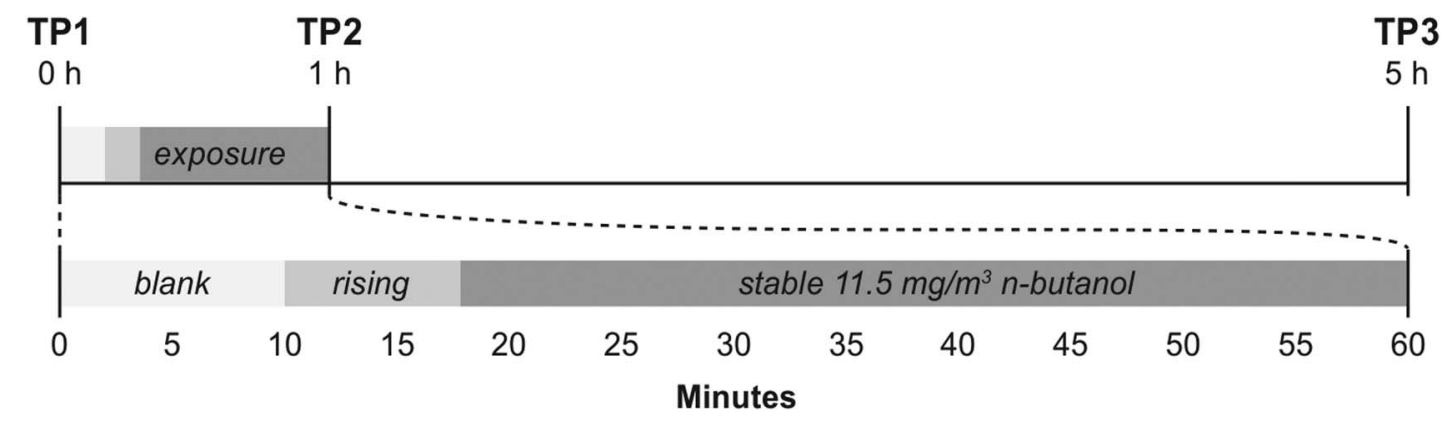

Figure 1 Overview of the exposure chamber procedure and sampling of blood. During the precondition, participants were seated in the exposure chamber with the door open. The door was thereafter closed, and the chamber session began at minute 0 . During the first $10 \mathrm{~min}$ of testing, no odorant was delivered into the chamber, after which $\mathrm{n}$-butanol was released into the chamber and reached a peak concentration after about $8 \mathrm{~min}$. The concentration remained at this peak level (3.7 ppm) for the remaining part of the exposure session. Venous blood samples was collected within 30 min prior to the exposure session (TP1), within 15 min postexposure session (TP2) and again 4 hours after the exposure session was terminated (TP3). TP, time point. 


\section{RNA isolation}

RNA was isolated using a modified RNeasy Micro Kit protocol (Qiagen, Valencia, California, USA). After thawing, white blood cells (WBCs) were harvested from $400 \mu \mathrm{L}$ buffy coat/RNAlater sample by centrifugation for $1 \mathrm{~min}$ at $14000 \mathrm{rpm}$ and the supernatant was subsequently discarded. WBCs were lysed by addition of $600 \mu \mathrm{L}$ RLT buffer (guanidine thiocyanate bufferQiagen, Valencia, California, USA) containing $10 \mu \mathrm{L}$ $\beta$-mercaptoethanol per $1 \mathrm{~mL}$ RLT buffer. After mixing for $15 \mathrm{~s}$ at $50 \mathrm{~Hz}$ in a tissue lyser (TissueLyser IIQiagen, Valencia, California, USA), $50 \mu \mathrm{L} 3 \mathrm{M}$ sodium acetate, $300 \mu \mathrm{L}$ acid phenol and $300 \mu \mathrm{L}$ chloroform were added, it was again mixed for $1 \mathrm{~min}$ at $50 \mathrm{~Hz}$ and subsequently centrifuged for $5 \mathrm{~min}$ at $14000 \mathrm{rpm}$. One millilitre ethanol and $200 \mu \mathrm{L}$ AVL buffer (Qiamp viral RNA mini kit, Qiagen, Valencia, California, USA) were added to the aqueous phase and mixed. The mixture was transferred to an RNeasy mini column, applied to vacuum on a QIAvac station (Qiagen, Valencia, California, USA) and washed with $350 \mu \mathrm{L}$ RW1 buffer (included in the RNeasy Micro Kit-Qiagen, Valencia, California, USA). Then, $80 \mu \mathrm{L}$ DNase I was added to the column, and the column with DNase I solution was incubated for $30 \mathrm{~min}$ at room temperature. Subsequently, the column was washed once with $350 \mu \mathrm{L}$ buffer RW1, two times with $700 \mu \mathrm{L}$ buffer RPE and finally with $700 \mu \mathrm{L} 80 \%$ ethanol. After drying the membrane, the column was transferred to a collection tube and RNA was eluted with $30 \mu \mathrm{L}$ RNase-free water by centrifugation for $1 \mathrm{~min}$ at $10000 \mathrm{rpm}$. RNA concentration and quality for each sample were determined as OD260/280 using a nanodrop spectrophotometer and for the integrity measurement, $1 \mu \mathrm{L}$ of the total RNA was analysed on an RNA 6000 nanochip using an Agilent Bioanalyzer. Total RNA concentrations were in the range of $14-231 \mu \mathrm{g} / \mathrm{uL}$ and the RNA integrity number was above 7.5 for all samples.

\section{cDNA synthesis}

cDNA was synthesised from $1.0 \mu \mathrm{g}$ total RNA in a $20 \mu \mathrm{L}$ reaction using QIAGEN RT ${ }^{2}$ First Strand Kit (catalog number 330401) designed and optimised gene expression analysis with QIAGEN RT ${ }^{2}$ Profiler PCR Arrays and RT $^{2}$ qPCR Primer Assays.

\section{Gene selection}

Genes included in the study either play a role in regulation of the common symptoms of MCS or in the physiological response observed during the exposure session, or they are involved in the proposed biological explanatory models of MCS. Additionally, as the non-specific symptoms in MCS somewhat resemble other unexplained disorders (eg, fibromyalgia and chronic fatigue syndrome), research findings into these disorders have been used as inspiration in selection of genes based on the possibility of a shared or overlapping pathophysiology. ${ }^{19} 244142$ All genes quantified are listed in table 1 with genes grouped into the biochemical pathways they are associated with, that is, immune regulation, sensory ion channel receptors, serotonin and receptors for neuromodulators, neural growth factor, the antioxidative enzyme, catalase, and the sphingosine-1-phosphate pathway. A short description of the motivation behind inclusion of each gene and reference to the relevant scientific literature is provided in online supplementary file 1 .

\section{RT2 Profiler PCR array}

Each 384-well (32 genes/12 samples) array contained 26 target genes, one reverse transcription control (NRT), one template control (NTC) and one positive PCR controls (PPC) as well as the following housekeeping genes (HKG); 18S ribosomal RNA (18SrRNA-Refseq\# X03205.1), ribosomal protein, large, P0 (RPLP0—Refseq\# NM_001002), hydroxymethylbilane synthase (HMBSRefseq\# NM_000190). Three hundred and eighty-four-well Custom RT2 Profiler PCR Arrays were performed according to the manufacturer's instructions (Qiagen, Valencia, California, USA) using the ABI prism 7900 HT (384-well format) instrument (Applied Biosystems, Foster City, California, USA) and ABI Prism 7900 SDS software V.2.1. Using a robotic work station, cDNA templates were mixed with ready-to-use RT2 qPCR Master Mixes and $10 \mu \mathrm{L}$ of the PCR component mix was aliquoted into each well containing predispensed gene-specific primer sets. Each plate was loaded with cDNA from 12 individual participants and all cDNA samples were run in duplicate on different arrays. Gene expression measured from each participant was normalised according to the average expression level of RPLP0 and HMBS in the same participant. The specificity of the SYBR Green assay was confirmed by melting point analysis.

\section{Statistical analysis}

The gene expression amounts were computed relative to HKG using the delta cycle threshold (dCT) method. The $2^{\wedge^{-\Delta c t}}$ values for all participants in each group were combined into a MCS or control group expression value that was used for group comparisons. Data are presented as group mean $\pm \mathrm{SD}$ and analysed using either Student's t-test or one-way analysis of variance with $\mathrm{p}<0.05$ considered to be statistically significant for each comparison. $p$ Values were subsequently adjusted for multiple testing by the Holm-Bonferroni method. ${ }^{43}$ In addition to the three TP comparisons, TP measures were also combined into a single area under the curve (AUC) value. For all candidate genes, AUC for each participant was computed by summing $2^{\wedge^{-\Delta c t}}$ values at TP1, TP2 and TP3 by trapezoidal integration and these values were then combined intro mean group AUC values. For statistical analysis, group $2^{\wedge^{-\Delta c t}}$ values and group AUC values were log-transformed. Principal component analysis (PCA) was performed on autoscaled $2^{\wedge-\Delta c t}$ values at TP1, TP2 and TP3 as well as on the AUC for expression of each gene during the exposure using the software package Latentix V.2.12 (http://www.latentix.com), and scores for principal component (PC)1, PC2 and PC3 were 
Table 1 Genes selected for quantitative expression analysis

\begin{tabular}{|c|c|c|c|c|}
\hline Class & Gene name & Acronym & Refseq \# & $\begin{array}{l}\text { Primer catalog } \\
\text { number* }^{*}\end{array}$ \\
\hline \multirow[t]{8}{*}{ Immune regulation } & Interleukin-1 $\beta$ & $\mathrm{IL}-1 \beta$ & NM_000576 & PPH00171C \\
\hline & Interleukin-2 & IL-2 & NM_000586 & PPH00172C \\
\hline & Interleukin-6 & IL-6 & NM_000600 & PPH00560C \\
\hline & Interleukin-8 & IL-8 & NM_000584 & PPH00568A \\
\hline & Interleukin-10 & $\mathrm{IL}-10$ & NM_000572 & PPH00572C \\
\hline & Tumour necrosis factor- $\alpha$ & TNF- $\alpha$ & NM_000594 & PPH00341F \\
\hline & Nitric oxide synthase 2 , inducible & NOS2 & NM_000625 & PPH00173F \\
\hline & $\begin{array}{l}\text { Nuclear factor of } \kappa \text { light polypeptide gene } \\
\text { enhancer in } B \text { cells } 1\end{array}$ & NFKB1 & NM_003998 & PPH00204F \\
\hline \multirow[t]{7}{*}{ Sensory ion channels } & $\begin{array}{l}\text { Purinergic receptor P2X, ligand-gated ion } \\
\text { channel, } 4\end{array}$ & $\mathrm{P} 2 \mathrm{R} \times 4$ & NM_002560 & PPH00341F \\
\hline & $\begin{array}{l}\text { Purinergic receptor P2X, ligand-gated ion } \\
\text { channel, } 5\end{array}$ & P2RX5 & NM_175081 & PPH19418A \\
\hline & $\begin{array}{l}\text { Transient receptor potential cation channel, } \\
\text { subfamily V, member } 1\end{array}$ & TRPV1 & NM_018727 & PPH08086F \\
\hline & $\begin{array}{l}\text { Transient receptor potential cation channel, } \\
\text { subfamily V, member } 4\end{array}$ & TRPV4 & NM_021625 & PPH16107B \\
\hline & $\begin{array}{l}\text { Transient receptor potential cation channel, } \\
\text { subfamily A, member } 1\end{array}$ & TRPA1 & NM_007332 & PPH12389E \\
\hline & Glutamate receptor, ionotropic, kainate 2 & GRIK2 & NM_021956 & PPH01863A \\
\hline & $\begin{array}{l}\text { Glutamate receptor, ionotropic, } N \text {-methyl } \\
\text { D-aspartate } 1\end{array}$ & GRIN1 & NM_007327 & PPH01823F \\
\hline \multirow{2}{*}{ Serotonin receptor } & 5-hydroxytryptamine (serotonin) receptor $1 \mathrm{~A}$ & HTR1A & NM_000524 & PPH02530E \\
\hline & 5-hydroxytryptamine (serotonin) receptor $2 \mathrm{~A}$ & HTR2A & NM_000621 & PPH01861G \\
\hline \multirow[t]{3}{*}{ Adrenergic receptors } & Adrenergic $\beta-1$ receptor & ADRB1 & NM_000684 & PPH02091B \\
\hline & Adrenergic $\beta-2$ receptor & ADRB2 & NM_000024 & PPH01856E \\
\hline & Catechol-O-methyltransferase & СОMT & NM_000754 & PPH01584B \\
\hline Substance $\mathrm{P}$ receptor & Tachykinin receptor 1 & TACR1 & NM_001058 & PPH01825A \\
\hline Nerve growth factor & Brain-derived neurotrophic factor & BDNF & NM_001709 & PPH00569F \\
\hline Antioxidative enzyme & Catalase & CAT & NM_001752 & PPH00420B \\
\hline \multirow[t]{3}{*}{$\begin{array}{l}\text { Sphingosine-1-phosphate } \\
\text { pathway }\end{array}$} & $\begin{array}{l}\mathrm{N} \text {-acylsphingosine amidohydrolase (acid } \\
\text { ceramidase) } 1\end{array}$ & ASAH1 & NM_004315 & PPH02492F \\
\hline & Sphingosine kinase 1 & SPHK1 & NM_021972 & PPH02491A \\
\hline & Sphingosine-1-phosphate lyase 1 & SGPL1 & NM_003901 & PPH13925A \\
\hline
\end{tabular}

plotted against each other to detect the otherwise hidden patterns in the data set.

\section{Software}

Excel was used to calculate the relative mRNA levels and calculate AUC values. Excel and Graphad Prims were used to create table and graphs, and multivariate PCA was performed using LatentiX V.2.11 (Latent5; http:// www. latentix.com)

\section{RESULTS}

\section{Participant characteristics}

A total of 36 participants participated in the study; 18 fulfilling the criteria for MCS and 18 enrolled as healthy controls. Blood samples from one control participant were lost during the RNA extraction procedure, leaving a control group of 17 participants. Selected characteristics of both study groups are shown in table 2. More women than men participated in the study and the mean age was
Table 2 Observed characteristics of MCS and the control group

\begin{tabular}{lllr}
\hline & $\begin{array}{l}\text { MCS group } \\
(\mathbf{n}=18)\end{array}$ & $\begin{array}{l}\text { Control } \\
\text { group } \\
(\mathbf{n}=17)\end{array}$ & p Value $^{*}$ \\
\hline Sex male/female, $\mathrm{n}$ & $2 / 16$ & $4 / 13$ & \\
Age mean ( \pm SD) & $44(14)$ & $40(14)$ & 0.447 \\
Chemical Sensitivity & $96(16)$ & $72(11)$ & $<0.001$ \\
Scale mean ( \pm SD) & & & \\
\hline *p Values refer to results of Mann-Whitney U test. \\
MCS, multiple chemical sensitivity.
\end{tabular}

comparable between the two groups. Compared with the controls, the MCS group showed a significantly higher score on the Chemical Sensitivity Scale ${ }^{36}(p<0.001)$. A more comprehensive characterisation of the study population can be found in Andersson et $a l^{30}$ showing a significantly higher score on the somatisation subscale of the 
symptom checklist (SCL) $90(\mathrm{p}<0.05)$ associated with MCS, and with no group differences for depression, anxiety and perceived stress. Likewise, the MCS group also reported higher number of morbidities other than MCS. No other significant demographic group differences were found.

\section{Gene expression levels}

Expression levels of multiple genes showed only limited transcriptional activity in leucocytes with cycle threshold (Ct) values above the predefined cut-off at 35 . In order to secure reliable expression data for the subsequent analysis, genes with average Ct value equal to or above 34 were excluded from the analysis. This was the case for the following genes; interleukin (IL)-2, glutamate receptor, ionotropic, kainate 2 , glutamate receptor, ionotropic, $N$-methyl D-aspartate 1 , adrenergic $\beta-1$ receptor, 5-hydroxytryptamine (serotonin) receptor 1A (HTR1A), HTR2A, nitric oxide synthase 2, tachykinin receptor 1 and brain-derived neurotrophic factor (see table 1 for additional gene information).

\section{Baseline gene expression levels}

No statistically significant group differences in gene expression were identified at baseline (TP1) for any of the 17 genes that were successfully quantified (figure 2). Likewise, PCA of the gene expression patterns revealed no differences between MCS participants and controls (data not shown).

\section{Gene expression time course}

MCS participantsperceived the n-butanol exposure as being more intense, more unpleasant and rated symptoms to be of greater magnitude compared with controls as reported in Andersson et al. ${ }^{30}$ Here, we examined whether the group differences in symptom elicitation after the n-butanol exposure were associated with in situ changes in expression rate of the target genes listed in table 1. Relative gene expression was monitored at three TPs and time-course graphs visualising mean expression levels of target genes IL-1 $\beta$, IL-6, IL-10, nuclear factor of $\kappa$ light polypeptide gene enhancer in $\mathrm{B}$ cells, transient receptor potential cation channel, subfamily $\mathrm{V}$, member 1 (TRPV1), TRPV4, catechol-O-methyltransferase (COMT) and $\mathrm{N}$-acylsphingosine amidohydrolase (acid ceramidase) 1 (ASAH1) are depicted in figure 3. Time-course graphs of the remaining nine target genes can be found in the online supplementary file 2 .

The comparable gene expression levels between the groups measured at baseline allowed for a direct comparison of gene expression levels between MCS participants and controls at TP2 and TP3, without prior adjustment. However, although visual inspection of several graphs indicates changes in gene transcription from TP1 to TP2 and/or TP3 for genes such as IL-10 and TRPV4, the differences were not statistically significant. Additionally, repeated blood sampling at three consecutive TPs allowed for analyses of time-dependent changes in gene expression levels taking place simultaneously in both groups as a result of the exposure. Yet, no statistically significant time-dependent changes in gene expression levels were found. A multivariate PCA was used to explore if the gene expression pattern differed in MCS participants compared with controls. However, the analysis did not reveal any statistically significant differences in score value at PC1, PC2 or PC3, between the two groups (data not shown).

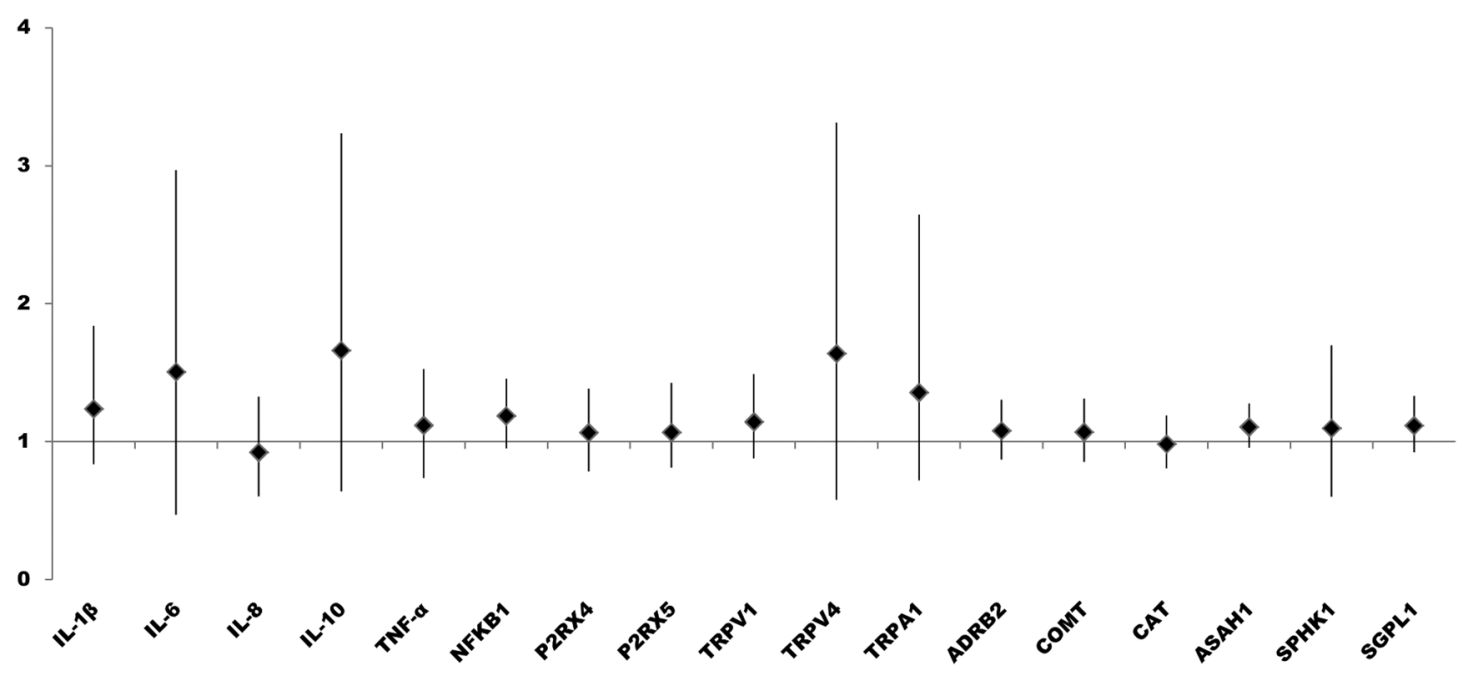

Figure 2 Ratios of relative gene expression in unexposed MCS versus healthy controls. Data represent ratios of mean with $95 \%$ Cls between gene expression levels in leucocytes from MCS participants and healthy controls. Full form of genes abbreviations are provided in table 1. ADRB2, adrenergic $\beta$-2 receptor; ASAH1, $N$-acylsphingosine amidohydrolase (acid ceramidase) 1; CAT, catalase; COMT, catechol-O-methyltransferase; IL, interleukin; MCS, multiple chemical sensitivity; NFKB1, nuclear factor of $\kappa$ light polypeptide gene enhancer in B cells 1; P2RX4, purinergic receptor P2X, ligand-gated ion channel, 4; P2RX5, purinergic receptor P2X, ligand-gated ion channel, 5; SGPL1, sphingosine-1-phosphate lyase 1; TNF, tumour necrosis factor; TRPA1, transient receptor potential cation channel, subfamily A, member 1; TRPV1, transient receptor potential cation channel, subfamily V, member 1; TRPV4, transient receptor potential cation channel, subfamily V, member 4. 
IL1ß

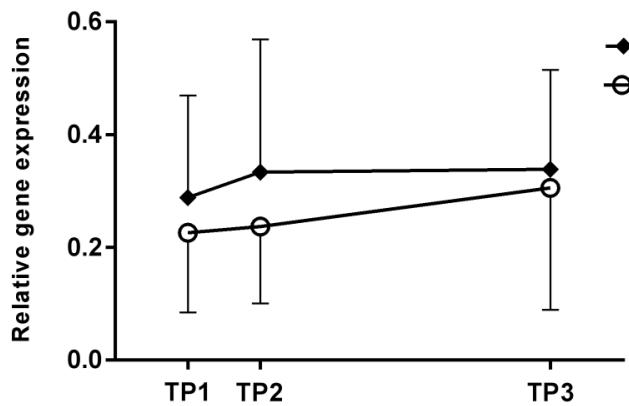

IL-10

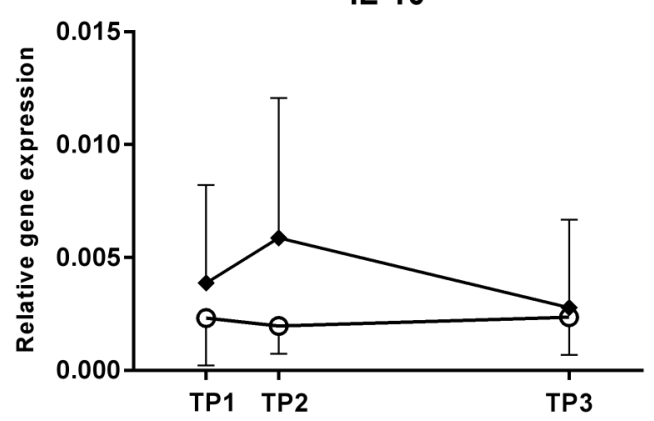

TRPV1

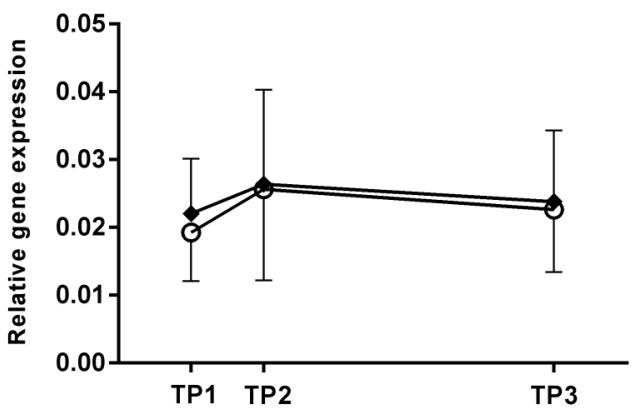

COMT

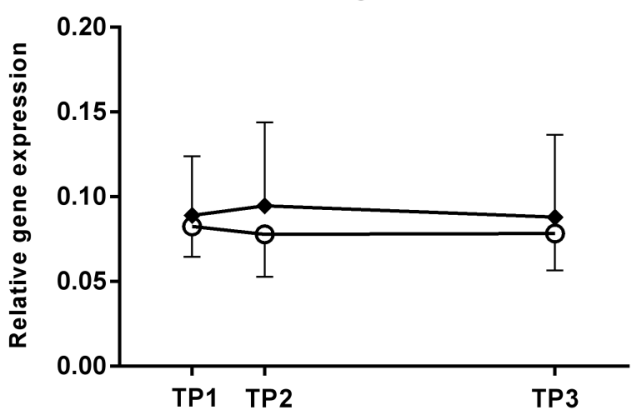

IL-6

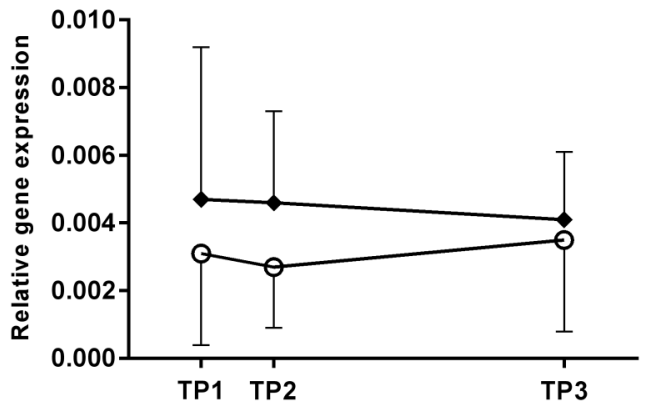

NFKß

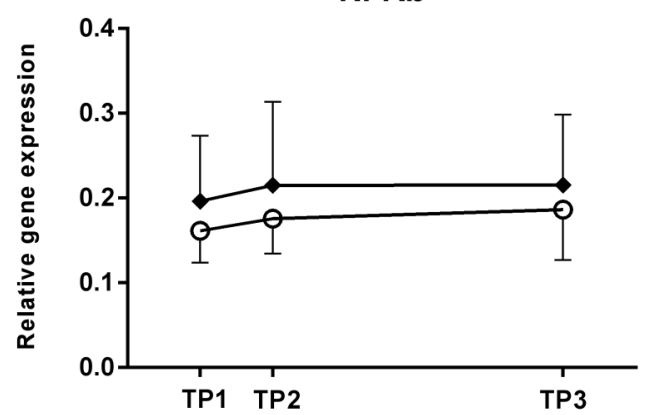

TRPV4

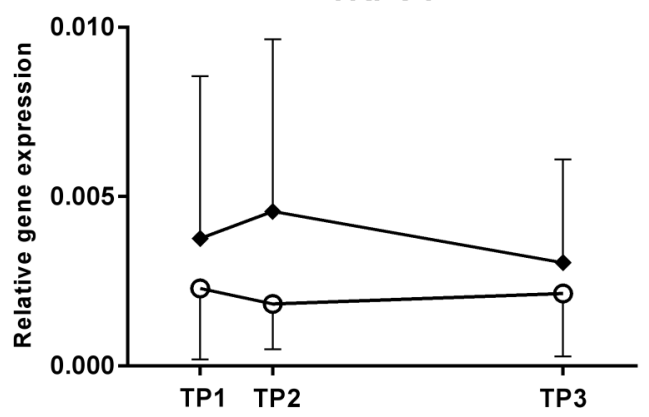

ASAH1

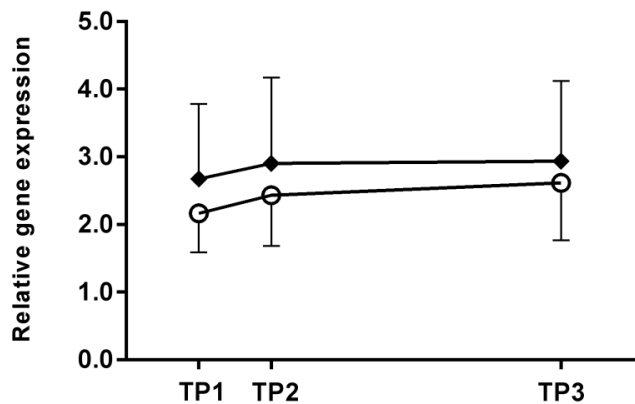

Figure 3 n-Butanol exposure and relative gene expression levels. Data are shown as mean levels with SD of IL-1 $\beta$, IL-6, IL-10, NFKB, TRPV1, TRPV4, COMT and ASAH1 quantified in buffy coat samples from the MCS participants and control. Data is presented at each of the three TPs of sampling: within 30 min prior to the exposure (TP1), within 15 min postexposure (TP2) and 4 hours after the exposure session had been terminated (TP3). ASAH1, $\mathrm{N}$-acylsphingosine amidohydrolase (acid ceramidase) 1; COMT, catechol-O-methyltransferase; IL, interleukin; MCS, multiple chemical sensitivity; NFKB1, nuclear factor of $\kappa$ light polypeptide gene enhancer in B cells 1; TP, time point; TRPV1, transient receptor potential cation channel, subfamily V, member 1; TRPV4, transient receptor potential cation channel, subfamily V, member 4. 


\section{AUC comparison}

After adjusting for multiple comparisons, we identified no significant AUC differences between MCS participants and controls. Based on single comparisons of gene expression, we did observe an increased AUC value for IL-6 in the MCS group (unadjusted $\mathrm{p}=0.03$ ), as well as a tendency towards increased AUC in the MCS group for IL-10 ( $p=0.074)$ and ASAH1 ( $p=0.092$; table 3). Generally, the AUC estimates revealed higher expression rates in the MCS group compared with controls, although not significant (table 3). Moreover, using PCA to explore the multivariate expression pattern of all successfully analysed genes did not reveal any statistical differences between the groups in the score values in either PC1, PC2 or PC3, nor in the three-dimensional space when the PCs were plotted against each other. PC1 explained the $\sim 40 \%$ variation in the data set, PC2 $\sim 15 \%$ and PC3 $\sim 11 \%$, see online supplementary file 3 .

\section{DISCUSSION}

This exploratory study used gene expression quantification for the first time to identify gene transcriptional changes in MCS participants upon a symptom eliciting n-butanol exposure session, when compared with controls. ${ }^{30}$ Overall our data demonstrated no statistically significant abnormalities in gene expression regulation in MCS participants at any of the three TPs. Nevertheless, exploration of the longitudinal responses to the exposure session represented by an integrated AUC value did reveal a tendency towards an overall increase in transcription rate associated with MCS, most notable for IL-6 (unadjusted $\mathrm{p}=0.03$ ). As depicted in table 2, more women than men participated in the study with a mean age in the 40s, which is in accordance with earlier reported characteristics of participants with MCS, ${ }^{23} 44$ and the Chemical Sensitivity Scale score was higher for the MCS group, indicating that the case criteria for inclusion were successful.

However, how to define and segregate MCS participants from healthy controls is a recurrent challenge in the field of MCS and many studies rely entirely on subjective information provided by the affected participants or clinicians. ${ }^{45}$ In this study, we confirmed the presence of a MCS phenotype using questionnaire data (ie, Chemical Sensitivity Scale, table 2) as well as objective physiological and subjective psychological measures collected during the exposure session, as depicted by Andersson et $a l^{30}$ The phenotypic response observed showed that over the course of the exposure session, the MCS group perceived the odour intensities of n-butanol as more intense and more unpleasant, and they reported more symptoms. Additionally, higher than normal pulse rate and lower than normal pulse rate variability were associated with the MCS group. ${ }^{30}$ Overall, the discrepancies between MCS participants and

Table 3 AUC values for each gene

\begin{tabular}{|c|c|c|c|c|c|c|}
\hline \multirow[b]{2}{*}{ Genes } & \multicolumn{2}{|l|}{ MCS group } & \multicolumn{2}{|c|}{ Control group } & \multirow[b]{2}{*}{ p Values* } & \multirow[b]{2}{*}{ Adjusted $p$ values $†$} \\
\hline & Mean AUC & \pm SD & Mean AUC & \pm SD & & \\
\hline IL-1 $\beta$ & 1.352 & 0.705 & 1.050 & 0.649 & 0.235 & 1.0 \\
\hline IL-6 & 0.018 & 0.009 & 0.012 & 0.006 & 0.030 & 0.45 \\
\hline IL-8 & 1.133 & 0.687 & 1.316 & 0.671 & 0.343 & 1.0 \\
\hline IL-10 & 0.025 & 0.036 & 0.009 & 0.004 & 0.074 & 1.0 \\
\hline TNF $\alpha$ & 0.302 & 0.189 & 0.260 & 0.085 & 0.494 & 1.0 \\
\hline NFKB1 & 0.865 & 0.321 & 0.714 & 0.160 & 0.105 & 1.0 \\
\hline $\mathrm{P} 2 \mathrm{R} \times 4$ & 0.455 & 0.217 & 0.374 & 0.104 & 0.206 & 1.0 \\
\hline P2RX5 & 0.597 & 0.217 & 0.584 & 0.290 & 0.794 & 1.0 \\
\hline TRPV1 & 0.102 & 0.031 & 0.095 & 0.036 & 0.593 & 1.0 \\
\hline TRPV4 & 0.016 & 0.019 & 0.008 & 0.005 & 0.129 & 1.0 \\
\hline TRPA1 & 0.010 & 0.006 & 0.008 & 0.005 & 0.385 & 1.0 \\
\hline ADRB2 & 1.022 & 0.332 & 1.006 & 0.330 & 0.781 & 1.0 \\
\hline COMT & 0.369 & 0.181 & 0.311 & 0.079 & 0.259 & 1.0 \\
\hline CAT & 6.314 & 2.154 & 6.527 & 2.619 & 0.900 & 1.0 \\
\hline ASAH1 & 11.904 & 4.297 & 9.657 & 2.561 & 0.092 & 1.0 \\
\hline SPHK1 & 0.101 & 0.066 & 0.080 & 0.030 & 0.269 & 1.0 \\
\hline SGPL1 & 0.598 & 0.171 & 0.544 & 0.136 & 0.350 & 1.0 \\
\hline
\end{tabular}

* $p$ Values calculated for each gene using Student's t-test.

tpValues after Holm-Bonferroni correction for multiple testing.

Values have been estimated using trapezoidal integration covering the 5-hour time span between initial and the last blood sample. AUC values are presented as group means with $\mathrm{SD}$.

ADRB2, adrenergic $\beta$-2 receptor; ASAH1, $N$-acylsphingosine amidohydrolase (acid ceramidase) 1; AUC, area under the curve; CAT, catalase; COMT, catechol-O-methyltransferase; IL, interleukin; MCS, multiple chemical sensitivity; NFKB1, nuclear factor of $\kappa$ light polypeptide gene enhancer in B cells 1; P2RX4, purinergic receptor P2X, ligand-gated ion channel, 4; P2RX5, purinergic receptor P2X, ligand-gated ion channel, 5; SGPL1, sphingosine-1-phosphate lyase 1; SPHK1, sphingosine kinase 1; TNF, tumour necrosis factor; TRPA1, transient receptor potential cation channel, subfamily A, member 1; TRPV1, transient receptor potential cation channel, subfamily V, member 1; TRPV4, transient receptor potential cation channel, subfamily $\mathrm{V}$, member 4. 
controls on the parameters presented above suggest that the case criteria for inclusion in the study were successful, which underpin the rationality of gene expression comparisons presented here. Nevertheless, although MCS participants were selected using common MCS criteria and the controls group is age and gender matched, unaccounted factors such as overall health status, lifestyle, socioeconomic status and personality traits among participants do have the potential to affect individual participant's gene expression levels and thereby the overall findings of the study. ${ }^{40}$ Consequently, when working with a poorly defined syndrome such as MCS, any study conclusions would benefit from being repeated in a larger case-control setup or even in a population-based study design.

Our first hypothesis was that symptom-free participants with MCS would have similar gene expression levels at baseline compared with age-matched and sex-matched healthy control group. This hypothesis was supported by the current results, as no significant group differences were observed at baseline (figure 2). The baseline measure is also in accordance with the finding described in Andersson et al, ${ }^{30}$ who found that prior to the exposure session while being seated inside the exposure chamber with the door open, ratings of symptoms and chemosensory perception were comparable between MCS participants and controls. Combined, these results suggest that MCS participants do not differ from controls during unexposed conditions in terms of transcript rates of the genes included. Therefore, any subsequent group-associated changes in gene expression can be attributed to the exposure session.

Our second hypothesis was that upon an n-butanol exposure session MCS participants and healthy controls would show differential gene-expression patterns. This was only moderately substantiated as our result did not demonstrate any abnormal gene regulation in WBC from MCS participants immediately after exposure (TP2), 4 hours later (TP3) or accumulated over the 5-hour time course. However, IL-6, IL-10 and ASAH1 did show a trend towards overall higher expression levels in MCS participants over the 5-hour time course (table 3). For IL-10, and to some degree TRPV4 and COMT, expression rates did rise at TP2 immediately after exposure (although not reaching significance) in the MCS group and subsequently declined indicative of transcriptional activation of those genes in response to the exposure paralleled with symptoms elicitation. Similar but more apparent findings have been observed in gene expression studies encompassing patients with chronic fatigue syndrome. ${ }^{27} 28$

Two overall observations were made that may have had pivotal influence on the study outcome. First, although group differences were not significant, the mean transcription rates were higher in the MCS group for all genes, except for IL-8 and catalase (figures 1 and 2, table 3 and see online supplementary file 2). Second, the intragroup variation was noticeably greater among
MCS participants than controls for all comparisons, even at baseline (figures 1 and 2, table 3 and see online supplementary file 2). The current data do not provide any definitive explanation for these observations. However, as depicted in Andersson et $a l^{30}$ the MCS group reported greater symptoms and rated the exposure session as more intense during the initial $10 \mathrm{~min}$ of blank exposure compared with controls. It was put forward that this MCS associated reaction to blank exposure was likely the result of increased expectancies, that is, MCS participants were aware that the exposure session was intended to induce symptom elicitation. Although speculative, negative expectancies may likewise have influenced gene expression levels among MCS participants from even prior to the exposure session, leading to nervous behaviour and increased stress levels. The magnitude of stress introduced and how the stress influences gene expression would also differ considerable among the exposed participants based on personality characteristics, which can explain, at least partially, the pronounced heterogeneity observed within the MCS group.

As emphasised earlier, future studies applying gene expression profiling in MCS research would benefit from either a more homogeneous study population, although such a study would only represent a fraction of the MCS community, or a higher number of participants, thereby providing a more representative platform for the analysis as well as increasing the statistical power. A large study population would also make subgroup analysis possible, taking into account the intragroup differences. Similarly, the inclusion of additional genes, for example, more immunological mediators, may have strengthened the study by providing a more comprehensive expressional characterisation. However, this was an exploratory study, and our results do suggest that gene expression characterisation of MCS participants on group levels is challenging. The genetic as well as epigenetic heterogeneity between MCS participants has been highlighted before, ${ }^{20}{ }^{46}$ and it has been recommended to divide MCS participants into subgroups of individuals stratified according to the symptom patterns or exposure agent reactivity. ${ }^{46} 47$ This strategy was not considered applicable with data from 18 individuals constituting the MCS group, as dividing participants into even smaller subgroups would have lowered the statistical power even further. Unfortunately, 36 participants was the maximum number of participants that systematically could be recruited, fulfilling the criteria, and completing the exposure procedure within the time and resources available. It could also have strengthened the study if a blank exposure sample in a blinded setup was included. Unfortunately, blood sample collection during the initial $10 \mathrm{~min}$ of blank exposure was not possible due to the design of the exposure chamber.

There exists a general consensus among clinicians and researchers that neurophysiological abnormalities play a vital role in MCS pathogenesis and both the neural and 
central sensitisation theories have been proposed as the driving mechanism in MCS. ${ }^{15} 24414849$ In light of the recent years advances made using electrophysiological outcome measures to analyse CNS activation/deactivation patterns among MCS participants during olfactory stimulation, it could be highly beneficial for future studies of gene expression in MCS upon symptoms elicitation to combine the current experimental setup with one of those technologies. Brain imaging technologies such as positron emission tomography, functional MRI and near-infrared spectroscopy have been used in a number of studies to detect altered reactions in the CNS of MCS participants upon olfactory stimulation..$^{50-55}$ By combining physiological phenotype measured during exposure ${ }^{30}$ with brain imaging during the exposure and WBC gene expression analysed in a multifaceted analysis, a more comprehensive model could be designed in an attempt to describe the underlying pathogenesis.

\section{CONCLUSION}

Collectively, our study did not reveal any statistically supported gene regulatory changes in MCS participants upon a symptom-eliciting exposure session using a low dose of the odorant n-butanol. MCS participants could therefore not be separated from controls based on gene expression analysed and we identified no correlation between regulation of specific genes and symptoms elicitation.

\section{Author affiliations}

${ }^{1}$ Danish Research Centre for Chemical Sensitivities, Copenhagen University Hospital, Gentofte, Denmark

${ }^{2}$ Department of Biotechnology and Biomedicine, Technical University of Denmark, Lyngby, Denmark

${ }^{3}$ Research Centre for Prevention and Health, Copenhagen, Denmark

${ }^{4}$ Department of Psychology, Umeå University, Umeå, Sweden

${ }^{5}$ Department of Occupational and Public Health Sciences, University of Gävle, Umeå, Sweden

${ }^{6}$ Department of Dermato-Allergology, The National Allergy Research Center, Copenhagen University Hospital Gentofte, Denmark

${ }^{7}$ Department of Economics, Swedish University of Agricultural Sciences, Uppsala, Sweden

Acknowledgements The authors thank all the patients who took part in the study as well as the nurse who assisted with blood sample collection.

Contributors TMD, SS, LA, A-SC, KE, SN and LIH designed the study and wrote the study protocol. TMD, SS, SN, LA and A-SC secured the necessary funding. LA, A-SC and NL managed the recruitment and screening of study participants and they also took care of all contact with the study participants. TMD, LA, A-SC and NL carryout the chemical exposure work and TMD were responsible for simultaneous sample collection and handling. TMD, LIH and $\mathrm{KE}$ were responsible for the laboratory and analytical work and undertook data and statistical analysis. Authors TMD and LIH, KE and SS interpreted the gene expression data. TMD, SS, LA and LIH managed the literature searches and wrote the first draft of the manuscript. All authors participated in writing the paper, reviewed it for important intellectual content and approved the final version.

Funding This study was supported by grants from the Swedish Council for Working Life and Social Research (2011-0396), the Danish Ministry of the Environment, and the Swedish Foundation for Humanities and Social Sciences (M14-0375:1).

Competing interests None declared.
Ethics approval All procedures performed in studies involving human participants were in accordance with the ethical standards of the institutional and/or national research committee and with the 1964 Helsinki declaration and its later amendments or comparable ethical standards and approved by the Ethics Committee at Umeå University (Dnr 2013-19-31). All participants were given written and oral information about the study. All participants were given 500 Swedish kronor ( $€ 50)$ for their participation.

Provenance and peer review Not commissioned; externally peer reviewed.

Data sharing statement Anonymised raw quantitative PCR data as well as any computed data can be obtained by contacting the first author, TMD by email: thomas.meinertz.dantoft@regionh.dk

Open Access This is an Open Access article distributed in accordance with the Creative Commons Attribution Non Commercial (CC BY-NC 4.0) license, which permits others to distribute, remix, adapt, build upon this work noncommercially, and license their derivative works on different terms, provided the original work is properly cited and the use is non-commercial. See: http:// creativecommons.org/licenses/by-nc/4.0/

\section{REFERENCES}

1. Berg ND, Linneberg A, Dirksen A, et al. Prevalence of self-reported symptoms and consequences related to inhalation of airborne chemicals in a Danish general population. Int Arch Occup Environ Health 2008;81:881-7.

2. Caress SM, Steinemann AC. Prevalence of multiple chemical sensitivities: a population-based study in the southeastern United States. Am J Public Health 2004;94:746-7.

3. Hausteiner C, Bornschein S, Hansen J, et al. Self-reported chemical sensitivity in Germany: a population-based survey. Int J Hyg Environ Health 2005;208:271-8.

4. Johansson A, Brämerson A, Millqvist E, et al. Prevalence and risk factors for self-reported odour intolerance: the Skövde population-based study. Int Arch Occup Environ Health 2005;78:559-64.

5. Miller CS, Prihoda TJ. The Environmental Exposure and Sensitivity Inventory (EESI): a standardized approach for measuring chemical intolerances for research and clinical applications. Toxicol Ind Health 1999;15:370-85.

6. Gibson PR, Elms AN, Ruding LA. Perceived treatment efficacy for conventional and alternative therapies reported by persons with multiple chemical sensitivity. Environ Health Perspect 2003;111:1498-504.

7. Skovbjerg S, Brorson S, Rasmussen A, et al. Impact of self-reported multiple chemical sensitivity on everyday life: a qualitative study. Scand J Public Health 2009;37:621-6.

8. Dantoft TM, Andersson L, Nordin S, et al. Chemical intolerance. Curr Rheumatol Rev 2015;11:167-84.

9. Consensus on Multiple Chemical Sensitivity. Multiple chemical sensitivity: a 1999 consensus. Arch Environ Health 1999;54:147-9.

10. Lacour M, Zunder T, Schmidtke K, et al. Multiple chemical sensitivity syndrome (MCS) - suggestions for an extension of the US MCS-case definition. Int J Hyg Environ Health 2005;208:141-51.

11. Graveling RA, Pilkington A, George JP, et al. A review of multiple chemical sensitivity. Occup Environ Med 1999;56:73-85.

12. Caress SM, Steinemann AC. A review of a two-phase population study of multiple chemical sensitivities. Environ Health Perspect 2003;111:1490-7.

13. Kreutzer R, Neutra RR, Lashuay N. Prevalence of people reporting sensitivities to chemicals in a population-based survey. Am $J$ Epidemiol 1999;150:1-12.

14. Park J, Knudson S. Medically unexplained physical symptoms. Health Rep 2007;18:43-7.

15. Sorg BA, Hochstatter T. Behavioral sensitization after repeated formaldehyde exposure in rats. Toxicol Ind Health 1999;15:346-55.

16. McKeown-Eyssen GE, Baines CJ, Marshall LM, et al. Multiple chemical sensitivity: discriminant validity of case definitions. Arch Environ Health 2001:56:406-12.

17. Ashford NA, Miller CS. Case definitions for multiple chemical sensitivity. In: National Research Council, ed. Multiple chemical sensitivities: a workshop. 1st edn. Washington DC: NATIONAL ACADEMY PRESS, 1992:41-5.

18. Winder C. Mechanisms of multiple chemical sensitivity. Toxicol Lett 2002;128:85-97.

19. Yunus MB. Fibromyalgia and overlapping disorders: the unifying concept of central sensitivity syndromes. Semin Arthritis Rheum 2007;36:339-56. 
20. Korkina L, Scordo MG, Deeva I, et al. The chemical defensive system in the pathobiology of idiopathic environment-associated diseases. Curr Drug Metab 2009;10:914-31.

21. Bailer J, Witthöft M, Paul C, et al. Evidence for overlap between idiopathic environmental intolerance and somatoform disorders. Psychosom Med 2005;67:921-9.

22. Pall ML. Multiple chemical sensitivity is a response to chemicals acting as toxicants via excessive NMDA activity. $J$ Psychosom Res 2010;69:327-8.

23. Das-Munshi J, Rubin GJ, Wessely S. Multiple chemical sensitivities: review. Curr Opin Otolaryngol Head Neck Surg 2007;15:274-80.

24. Yunus MB. Editorial review: an update on central sensitivity syndromes and the issues of nosology and psychobiology. Curr Rheumatol Rev 2015;11:70-85.

25. Gibson PR, Kovach S, Lupfer A. Unmet health care needs for persons with environmental sensitivity. $J$ Multidiscip Healthc 2015;8:59-66.

26. Gibson PR, Lockaby SD, Bryant JM. Experiences of persons with multiple chemical sensitivity with mental health providers. $J$ Multidiscip Healthc 2016;9:163-72.

27. White AT, Light AR, Hughen RW, et al. Differences in metabolitedetecting, adrenergic, and immune gene expression after moderate exercise in patients with chronic fatigue syndrome, patients with multiple sclerosis, and healthy controls. Psychosom Med 2012;74:46-54.

28. Light AR, Bateman L, Jo D, et al. Gene expression alterations at baseline and following moderate exercise in patients with Chronic Fatigue Syndrome and Fibromyalgia Syndrome. J Intern Med 2012;271:64-81.

29. Light AR, White AT, Hughen RW, et al. Moderate exercise increases expression for sensory, adrenergic, and immune genes in chronic fatigue syndrome patients but not in normal subjects. $J$ Pain 2009;10:1099-112.

30. Andersson L, Claeson AS, Dantoft TM, et al. Chemosensory perception, symptoms and autonomic responses during chemical exposure in multiple chemical sensitivity. Int Arch Occup Environ Health 2016;89:79-88.

31. Dantoft TM, Skovbjerg S, Andersson L, et al. Inflammatory mediator profiling of n-butanol exposed upper airways in individuals with multiple chemical sensitivity. PLOS ONE 2015;10:e0143534.

32. Dantoft TM, Elberling J, Brix S, et al. An elevated proinflammatory cytokine profile in multiple chemical sensitivity. Psychoneuroendocrinology 2014;40:140-50.

33. De Luca C, Scordo MG, Cesareo E, et al. Biological definition of multiple chemical sensitivity from redox state and cytokine profiling and not from polymorphisms of xenobiotic-metabolizing enzymes. Toxicol Appl Pharmacol 2010;248:285-92.

34. Staud R. Cytokine and immune system abnormalities in fibromyalgia and other central sensitivity syndromes. Curr Rheumatol Rev 2015;11:109-15.

35. Cain WS. Testing olfaction in a clinical setting. Ear Nose Throat $J$ 1989;68(4):316, 322-16, 328

36. Nordin S, Millqvist E, Löwhagen $\mathrm{O}$, et al. The chemical sensitivity scale: psychometric properties and comparison with the noise sensitivity scale. J Environ Psychol 2003;23:359-67.
37. Andersson L, Claeson AS, Ledin L, et al. The influence of health-risk perception and distress on reactions to low-level chemical exposure. Front Psychol 2013;4:816

38. Van Thriel C, Haumann K, Kiesswetter E, et al. Time courses of sensory irritations due to 2-butanone and ethyl benzene exposure: influences of self-reported multiple chemical sensitivity (sMCS). Int $J$ Hyg Environ Health 2002;204:367-9.

39. Nagata Y. Measurement of Odor threshold by Triangle Odor Bag Method. J Jpn Air Cleaning Ass 2003;41:17-25.

40. Ruth $\mathrm{JH}$. Odor thresholds and irritation levels of several chemical substances: a review. Am Ind Hyg Assoc J 1986;47:A142-51.

41. Yunus MB. Central sensitivity syndromes: a new paradigm and group nosology for fibromyalgia and overlapping conditions, and the related issue of disease versus illness. Semin Arthritis Rheum 2008;37:339-52.

42. Schottenfeld RS, Cullen MR. Recognition of occupation-induced posttraumatic stress disorders. J Occup Med 1986;28:365-9.

43. Holm S. A simple sequentially rejective multiple test procedure. Scand J Stat 1979;6:65-70.

44. Gibson PR. Life indicators, illness characteristics, and psychosocial concomitants of self-reported multiple chemical sensitivity: A two-year longitudinal study. J Nurs Educ Pract 2014;4:204-16.

45. Kreutzer R. Idiopathic environmental intolerance: case definition issues. Occup Med 2000;15:511-17.

46. Berg ND, Rasmussen HB, Linneberg A, et al. Genetic susceptibility factors for multiple chemical sensitivity revisited. Int J Hyg Environ Health 2010;213:131-9.

47. NICNAS. Multiple chemical sensitivity: identifying key research needs; National Industrial Chemicals Notification and Assessment Scheme, Australia; Office of Chemical Safety and Environmental Health, Australia. Canberra, Australia: OCSEH, 2010.

48. Bell IR, Baldwin CM, Schwartz GE. Sensitization studies in chemically intolerant individuals: implications for individual difference research. Ann N Y Acad Sci 2001;933:38-47:38-47.

49. Bell IR, Baldwin CM, Fernandez M, et al. Neural sensitization model for multiple chemical sensitivity: overview of theory and empirical evidence. Toxicol Ind Health 1999;15:295-304.

50. Andersson L, Claeson AS, Nyberg L, et al. Brain responses to olfactory and trigeminal exposure in idiopathic environmental illness (IEI) attributed to smells-an fMRI study. J Psychosom Res 2014;77:401-8.

51. Hillert $\mathrm{L}$, Jovanovic $\mathrm{H}$, Ahs $\mathrm{F}$, et al. Women with multiple chemical sensitivity have increased harm avoidance and reduced 5-HT(1A) receptor binding potential in the anterior cingulate and amygdala. PLOS ONE 2013;8:e54781.

52. Hillert L, Musabasic $\mathrm{V}$, Berglund $\mathrm{H}$, et al. Odor processing in multiple chemical sensitivity. Hum Brain Mapp 2007;28:172-82.

53. Orriols R, Costa R, Cuberas $\mathrm{G}$, et al. Brain dysfunction in multiple chemical sensitivity. J Neurol Sci 2009;287:72-8.

54. Azuma K, Uchiyama I, Tanigawa M, et al. Assessment of cerebral blood flow in patients with multiple chemical sensitivity using near-infrared spectroscopy-recovery after olfactory stimulation: a case-control study. Environ Health Prev Med 2015;20:185-94.

55. Chiaravalloti A, Pagani M, Micarelli A, et al. Cortical activity during olfactory stimulation in multiple chemical sensitivity: a (18)F-FDG PET/CT study. Eur J Nucl Med Mol Imaging 2015;42:733-40. 